\title{
Do EEG-Biometric Templates Threaten User Privacy?
}

\author{
Full Paper
}

\author{
Yvonne Höller \\ Department of Neurology, Paracelsus Medical University \\ Salzburg \\ Salzburg, Austria \\ yvonne.hoeller@sbg.ac.at
}

\author{
Andreas Uhl \\ Department of Computer Sciences, University of Salzburg \\ Salzburg, Austria \\ uhl@cosy.sbg.ac.at
}

\begin{abstract}
The electroencephalogram (EEG) was introduced as a method for the generation of biometric templates. So far, most research focused on the optimisation of the enrolment and authentication, and it was claimed that the EEG has many advantages. However, it was never assessed whether the biometric templates obtained from the EEG contain sensitive information about the enrolled users. In this work we ask whether we can infer personal characteristics such as age, sex, or informations about neurological disorders from these templates.

To this end, we extracted a set of 16 feature vectors from EEG epochs from a sample of 60 healthy subjects and neurological patients. One of these features was the classical power spectrum, while the other 15 features were derived from a multivariate autoregressive model, considering also interdependencies of EEG channels. We classified the sample by sex, neurological diagnoses, age, atrophy of the brain, and intake of neurological drugs.

We obtained classification accuracies of up to .70 for sex, .86 for the classification of epilepsy vs. other populations, .81 for the differentiation of young vs. old people's templates, and .82 for the intake of medication targeted to the central nervous system. These informations represent privacy sensitive information about the users, so that our results emphasise the need to apply protective safeguards in the deployment of EEG biometric systems.
\end{abstract}

\section{CCS CONCEPTS}

\section{- Security and privacy $\rightarrow$ Privacy protections;}

\section{KEYWORDS}

EEG-Biometrics; user privacy; multivariate autoregressive model

\section{ACM Reference Format:}

Yvonne Höller and Andreas Uhl. 2018. Do EEG-Biometric Templates Threaten User Privacy?: Full Paper. In IH\&MMSec '18: 6th ACM Workshop on Information Hiding and Multimedia Security, June 20-22, 2018, Innsbruck, Austria. ACM, New York, NY, USA, 10 pages. https://doi.org/10.1145/3206004.3206006

Permission to make digital or hard copies of part or all of this work for personal or classroom use is granted without fee provided that copies are not made or distributed for profit or commercial advantage and that copies bear this notice and the full citation on the first page. Copyrights for third-party components of this work must be honored. For all other uses, contact the owner/author(s).

IH\&MMSec '18, June 20-22, 2018, Innsbruck, Austria

(c) 2018 Copyright held by the owner/author(s).

ACM ISBN 978-1-4503-5625-1/18/06.

https://doi.org/10.1145/3206004.3206006

\section{INTRODUCTION}

The electroencephalogram (EEG) is a neurological examination method used for clinical purposes, such as the diagnosis of epilepsy [45], the monitoring of anaesthesia [31], or the diagnosis of brain death [55], among others. Clinical evaluation of the EEG exists since Hans Berger has established the EEG as an assessment tool of brain activity in humans the 1930ies. This approach is based on qualitative assessment of the raw signal, that allows to examine the presence of natural rhythms such as the alpha rhythm at $8-12 \mathrm{~Hz}$ (Figure 1, left), and the detection of epileptiform phenomena such as polyspikes (Figure 1, middle). Qualitative EEG can be used in order to detect diffuse or focal pathological changes, by means of slowing of the background rhythm from the $\alpha(8-12 \mathrm{~Hz})$ to $\delta-\theta$ range $(0-7$ $\mathrm{Hz})[8,24]$. This slowing is regionally limited for focal pathologies such as brain tumors [22], transient or permanent ischemia [59], e.g. resulting from stroke, brain hemorrhage, traumatic injury, malformations of cortical development, focal epileptic spikes, neurodegeneration, arteriovenous malformations, and infections such as bacterial cerebritis or viral encephalitis [8, 24]. Generalized slowing can be found under medication with certain drugs [48], neurogenerative disorders, neurodevelopmental pathologies, hydrocephalus, metabolic or toxic encephalopathy, CNS infection disorders, and certain brain lesions [8, 24]. However, the EEG cannot be used in order to determine individual aspects such as sex, age, or in order to differentially classify pathological aspects, such as dementia.

In contrast, quantitative analysis of the EEG has emerged alongside with digital signal processing [37], where the raw signal is processed to form feature vectors such as spectral frequency decomposition or multivariate autoregressive modelling. In this field, the EEG has been shown to yield significant information for the early differential diagnosis of dementia [32].

Today, the EEG is being perceived as a promising technique to generate biometric templates, because the measured activity of the brain is universal, can not easily be circumvent, and there are also studies that evidenciate uniqueness and permanence of specific features extracted from the EEG $[1,4,7,11-14,35,43,53$, 63]. Moreover, modern mobile systems are small and cheap, so that the biometric characteristic can be captured quickly and easily $[23,33]$. EEG biometric features can be captured during rest, or during stimulation or cognitive effort [5, 38, 39, 41, 52, 54, 64, 65].

Qualitative review of the generated templates might not allow to draw any conclusions about characteristics of the investigated individual, since EEG experts are not trained to read such a representation of the information contained in the EEG, in contrast 

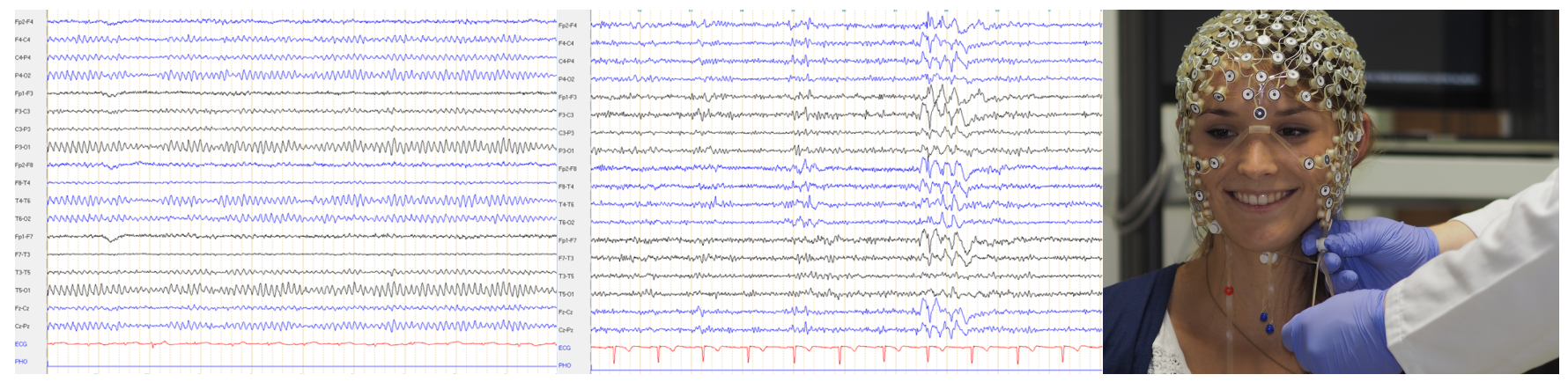

Figure 1: Can we extract informations about sex, age, and health from electroencephalographic biometric templates?

to their well-trained eye for raw EEG signals. Nevertheless, quantitative analysis of the templates is likely to reveal information about factors pertinent to the individuals, such as age, sex, and pathology. The EEG is highly indicative for epilepsy [42], and as for many other biometric characteristics, the EEG is heavily affected by healthy and pathological ageing [46]. For example, there are specific expectations of changes for sub-bands of EEG coherence [60]. In addition, sex affects the EEG [61, 62], and also the menstrual cycle in women [9].

In this work, we aim to demonstrate that it is technically possible to extract privacy sensitive information form EEG biometric templates. To this end, we classified EEG biometric templates by sex, age, diagnosis of neurological diseases, the intake of medication that affects the central nervous system, and neuroradiological pathological findings in the memory region hippocampus of the brain. The neurological diseases included in the analyses were patients with subjective cognitive complaints (SCC) or with mild cognitive impairment (MCI), and temporal lobe epilepsy (TLE). We included also healthy controls (HC), in order to compare them to the neurological populations. Both, SCI and $\mathrm{MCI}$ are claimed to be a prodromal phase of Alzheimer's disease, with conversion rates from MCI to Alzheimer's disease of up to 70\% [46]. TLE is a disease with medically uncontrollable and unpredictable seizures, therefore affecting all aspects of life. Nevertheless, the disease cannot be recognized unless one witnesses a seizure or performs qualitative analysis of an EEG with epileptiform activity. The burden and stigma of TLE is significant, so that patients try to keep the diagnosis confidential.

The paper is structured as follows: In Section 2 we describe which features we extracted from the EEG, what scenarios of templates and EEG data we examined, how we performed feature subset selection and classification, which informations we tried to extract, and finally, which sample data set we used. In Section 3 we present the results and Section 4 concludes the work.

\section{METHODS}

\subsection{Feature extraction}

We used a data sample as described in Section 2.7 with resting EEG recordings and 27 sensors.

We estimated power spectral density (PSD) as the single-sided amplitude spectrum from the Fast Fourier Transform of the signal.

Furthermore, we estimated autoregressive coefficients, from which we extracted a set of 14 measures of interaction between all of the 27 selected sensors. The estimation was based on the multivariate autoregressive model (MVAR) [19, 29]:

$$
Y(t)=\sum_{k=1}^{P} A(k) Y(t-k)+U(t)
$$

where $Y(t)=\left[y_{1}(t), \ldots, y_{M}(t)\right]^{T}$ is a vector holding the values of the $M$ channels at time $t, P$ is the model order, $A(k)$ are $M \times M$ coefficient matrices in which the element $a_{i j}(k)$ describes the dependence of $y_{i}(t)$ on $y_{j}(t-k)$ and $U(n)$ is the innovation process, which is assumed to be composed of white and uncorrelated noise. We used the functions mvfreqz.m and mvar.m from the BioSig toolbox [50] with model order $P=41$. The model order was chosen in order to obtain a large ratio $N /(M \cdot P)$ which is needed to get an accurate model estimation [49]. In this study, this resulted in a ratio of $20.5 \cdot 500 /(27 \cdot 41)=9.26$, which is well above an acceptable threshold. In order to estimate the multivariate autoregressive model we used partial correlation estimation with unbiased covariance estimates [29], which was found to be the most accurate estimation method according to [49].

Thus, the matrices $A(k)$ of size $M \times M$ formed the second feature, representing the multivariate autoregressive coefficients (AR).

The other 14 features were obtained as follows. The estimated MVAR model was transformed from the time-domain into the $z$ domain and the $f$-domain, which yields accordingly two transfer functions. The multivariate parameters in the frequency domain that can be derived from these transfer functions were computed for $1 \mathrm{~Hz}$ frequency steps between 1 and $125 \mathrm{~Hz}$.

- Direct causality (DC): Direct causality was developed by [21] to overcome the problem that the directed transfer function does not distinguish between direct and indirect information flows. Direct causality is the only measure that is not computed for each frequency.

- Spectrum (S): This contains the auto- and the cross-spectrum, which is the Fourier transform of the cross-covariance function [34]

- Transfer function (hh): This transfer function is related to the non-normalized directed transfer function [15].

- Transfer function polynomial (AF): This is the frequency transform of a polynomial describing the transfer function. The absolute of the squared transfer function polynomial is the non-normalized partial directed coherence [15]. 
- Real valued coherence ( $\mathrm{COH})$ : By considering the real part of the complex-valued coherence [36], the result is an ordinary coherence [50]. We will refer to it as coherence.

- Complex coherence (iCOH): By considering the imaginary part of the complex-valued coherence [36], we get complex coherence.

- Partial coherence (pCOH): This is the partial coherence, calculated with an alternative method as provided in the biosig-toolbox. Partial coherence, also known as Gersch causality, was first designed to identify epileptic foci by [17] The authors proposed that one channel is said to drive the other channels if the first channel explains or accounts for the linear relation between the other two. The real part of the partial coherence was used.

- Partial directed coherence (PDC): Partial directed coherence as an extended concept of partialized coherence, is a measure of the relative strength of the direct interaction between pairs of regions [2].

- Partial directed coherence factor (PDCF): The partial directed coherence factor [2] is an intermediate step between partial coherence and partial directed coherence. It adds directionality to partial coherence, but includes instantaneous causality, which is undesirable when examining processes that evolve over time like an epileptic seizure [51].

- Generalized partial directed coherence (GPDC): The major advantage of generalized partial directed coherence [3] over partial directed coherence is its robustness against scaling differences between the signals [57].

- Directed transfer function (DTF): Like directed coherence, directed transfer function represents information that flows from one region to another over many possible alternative pathways [20].

- Direct directed transfer function (dDTF): The direct directed transfer function extends the concept of directed transfer function by distinguishing between direct and indirect causal relations of signals [25]. As such, the concepts of partial coherence and directed transfer function are combined.

- full frequency directed transfer function (ffDTF): The difference between the directed transfer function and the full frequency directed transfer function [25] is that the directed transfer function is normalized by the total frequency content of the considered frequency band, while the full frequency directed transfer function is normalized with respect to all the frequencies in the predefined frequency interval. As such, the full frequency directed transfer function priorizes those frequencies which contribute the most to the power of the signal [58].

- Geweke's Granger Causality (GGC): This is a modified version of Geweke's Granger Causality [18], concretely the bivariate version as in [6].

Next, all frequency-dependent measures of interaction, that is, all but DC, and the PSD were averaged in classical frequency ranges delta $(\delta, 2-4 \mathrm{~Hz})$, theta $(\theta, 5-7 \mathrm{~Hz})$, alpha $(\alpha, 8-13 \mathrm{~Hz})$, beta $(\beta, 14-30$ $\mathrm{Hz})$, and gamma $(\gamma, 31-80 \mathrm{~Hz})$.

All frequency dependent measures were analyzed once with this 5-band frequency configuration, and once when including only the
3 frequency ranges $\theta, \alpha$, and $\beta$, since this range has been shown to be more informative by Maiorana et al. [27]. The non-frequency dependent measures DC and AR were calculated on the band-pass filtered data $(5-30 \mathrm{~Hz})$ for this purpose.

\subsection{Feature fusion}

The autoregressive coefficients were obtained as $27 \times 27$ matrices for each $k=1 \ldots P$ where $\mathrm{P}$ is the model order, in our case $P=41$. We concatenated these values as one long feature vector, i.e. all $27 \times 27 \times 41$ coefficients. For PSD, we concatenated the values from all 27 electrodes from all 5 frequency bands, thus resulting in $27 \times 5$ values in the feature vector. For each of the 14 measures derived from the autoregressive model, we obtained interaction matrices of size $27 \times 27$, thus, one value for each electrode combination. Depending on whether the value was frequency specific (all measures but DC) or not (DC), these interaction matrices were available separately for each of the 5 frequency ranges. Depending on whether the measure was directed or not, this matrix was symmetric (not-directed measures, e.g. coherence) and thus redundant or not symmetric (directed measures, e.g. directed transfer function). We concatenated all of the non-redundant values from these interaction matrices for all frequencies of interest. For nondirected measures we took the upper triangular of the interaction matrix and concatenated these values for each frequency range. For measures without time-lagged auto-correlation, the diagonal of the interaction matrix was excluded because it contained no information. This resulted in high-dimensional feature vectors of lengths ranging from $27 \times 27=729$ (DC as the only measure without frequency dimension) to $27 \times 27 \times 5=3645$ (all directed measures with autocorrelation).

\subsection{Single epochs and templates}

We tested the information content in the feature vectors from the single epochs and templates. A single epoch is a segment of the signal, in our experimental data of 20.5 seconds length. A template consists of the average feature vector calculated for several equal sized epochs $e_{1} \ldots e_{k}$ that belong to one subject $p$. Thus, one element $i$ in the template feature vector of subject $p$ was obtained as

$$
\text { template }_{p, i}=1 / k \sum_{l=1}^{k} z\left(\text { feat }_{p, e_{l}, i}\right)
$$

We obtained templates as the average of 3 epochs.

We performed the experiment with the following configurations:

(1) single epochs

(a) single epochs EEG session 1

(b) single epochs EEG session 2

(c) single epochs from both EEG sessions

(2) templates

(a) templates from EEG session 1

(b) templates from EEG session 2

(c) templates from both EEG sessions

We computed receiver operating characteristic (ROC) curve, the area under the curve (AUC) alongside with 95\% confidence intervals on the true positive rate by threshold averaging and sampling using bootstrap and 1000 replicas. 


\subsection{Classification}

We used support vector machines for classification, because they deal with non-linear properties of the data even when a linear kernel is used. When data are only non-linearly separable, the data is mapped into a feature space in which the linear separating hyperplane can be used. We performed a classification in the sense of supervised learning with a linear kernel function (dot product) and quadratic programming in order to find the separating hyperplane, resulting in a 2-norm soft-margin support vector machine, by using the matlab functions svmtrain and svmclassify from the statistics and machine learning toolbox.

\subsection{Feature selection algorithm}

The high-dimensional vectors are likely to contain redundant information, since neighbouring frequencies and neighbouring electrodes are likely to share information. We implemented a feature subset selection algorithm in a three-layered cross-validation procedure. With three layers the optimization is not done towards the test data population and thus, increases the generalizability of the result. The procedure, illustrated in Figure 2, was as follows:

(1) In the outer layer, we randomly partitioned the set of epochs or templates from 60 subjects into 5 sets for a 5 -fold crossvalidation. Thus, in 5 iterations, each time the epochs/templates of $20 \%$ of the subjects were left out as the test set, the other $80 \%$ are submitted to the middle layer as the training set.

(2) In the middle layer, the included subjects were again divided into 5 subsets, for a 5 -fold cross-validation. Thus, in 5 iterations, each time $20 \%$ of the subjects were left out as the left-out set, the other $80 \%$ were submitted to the inner layer as the optimization set.

(3) In the inner layer, the subjects that formed the optimization set were used in order to optimize the feature vector as follows:

(a) A $t$-test was calculated between the two groups.

(b) The resulting $p$-values were sorted in ascending order.

(c) The feature vector was initiated by taking the feature with the smallest $p$-value, thus, the initial length of the feature vector was one.

(d) For this feature vector, the classification accuracy was calculated with 5 -fold cross-validation, i.e. a model was trained 5 times with a model-generating set, and the accuracy was determined in each iteration by the respective validation set.

(e) Then, the next feature from the sorted list was added. For this feature vector, the classification with 5 -fold crossvalidation was repeated.

(f) The resulting accuracy for the feature vector with two entries was compared to the previous result of a feature vector with the first entry. The added entry to the feature vector was included only if the condition constraints were met as follows:

- the resulting classification accuracy was at least as high as the maximum of the previously obtained classification accuracies; that is, the second accuracy had to be larger than the first entry, or the 6th accuracy had to be larger than each of the five previous classification accuracies.

- If the so far best sensitivity/specificity, or in other words, accuracy for members of the first/second group, respectively, was lower than 0.75 , then the obtained sensitivity had to be at least as large as this maximum.

- If the so far best specificity/sensitivity, was lower than 0.5 , then the obtained specificity had to be larger than this maximum.

(g) This way, features were added and tested for their contribution to the classification accuracy until all available features were used, or until the feature vector reached a maximum of 30 entries, or if more than a consecutive number of 200 features was not added to the feature vector. We limited the maximally acceptable length of the feature vector to 30 entries in the inner loop of the feature vector optimization process, and 31 entries as a final selection.

(4) The average length $N$ of the resulting 5 optimised feature sets was calculated. The occurrences of the features across the 5 sets was counted. A final feature vector was formed by including only those features which were selected at least in 2 of the 5 iterations. If this resulted in no features, all features were included that were selected at least in 1 out of 5 iterations. If the resulting feature vector included more than $N$ features, only the top-most $N$ selected features were included.

(5) The resulting feature vector was used in order to train a support vector machine on the outer-layer training set, and the resulting model was used to classify the outer-layer test set. From the 5 classifications, the general classification accuracy and the within-group accuracy for the two groups (i.e. sensitivity/specificity) were determined.

The threshold of 0.75 was selected as rough estimator for abovechance classification; a value of 0.75 can be considered to be clearly above chance, since the expected chance level would be around 0.5 .

Please note again that the separation into training, evaluation and test set was done subject wise, so that the multiple epochs or templates from one subject were always all included into one of these three sets. That is, we had 6 or 12 epochs per subject when single epochs were classified, and 2 or 4 templates when the templates were classified. Moreover, all subsets were drawn in order to maintain the original proportion of the two groups, but the composition of the 5 subsets for the 5 -fold cross validation was obtained by randomized assignment of the subjects to the 5 subsets.

Sorting the features initially by the $p$-values is a trivial but nevertheless efficient way to shorten the time-consuming step of a wrapper-style feature subset selection procedure. Please note that similar approaches implemented principal component analysis [40, 44], independent component analysis [56], or benchmarked the feature selection according to principal component analysis by the ratio of within-subject to between-subject variability [28].

\subsection{Classifications}

We examined the following pairwise classification scenarios:

sex female vs. male subjects 


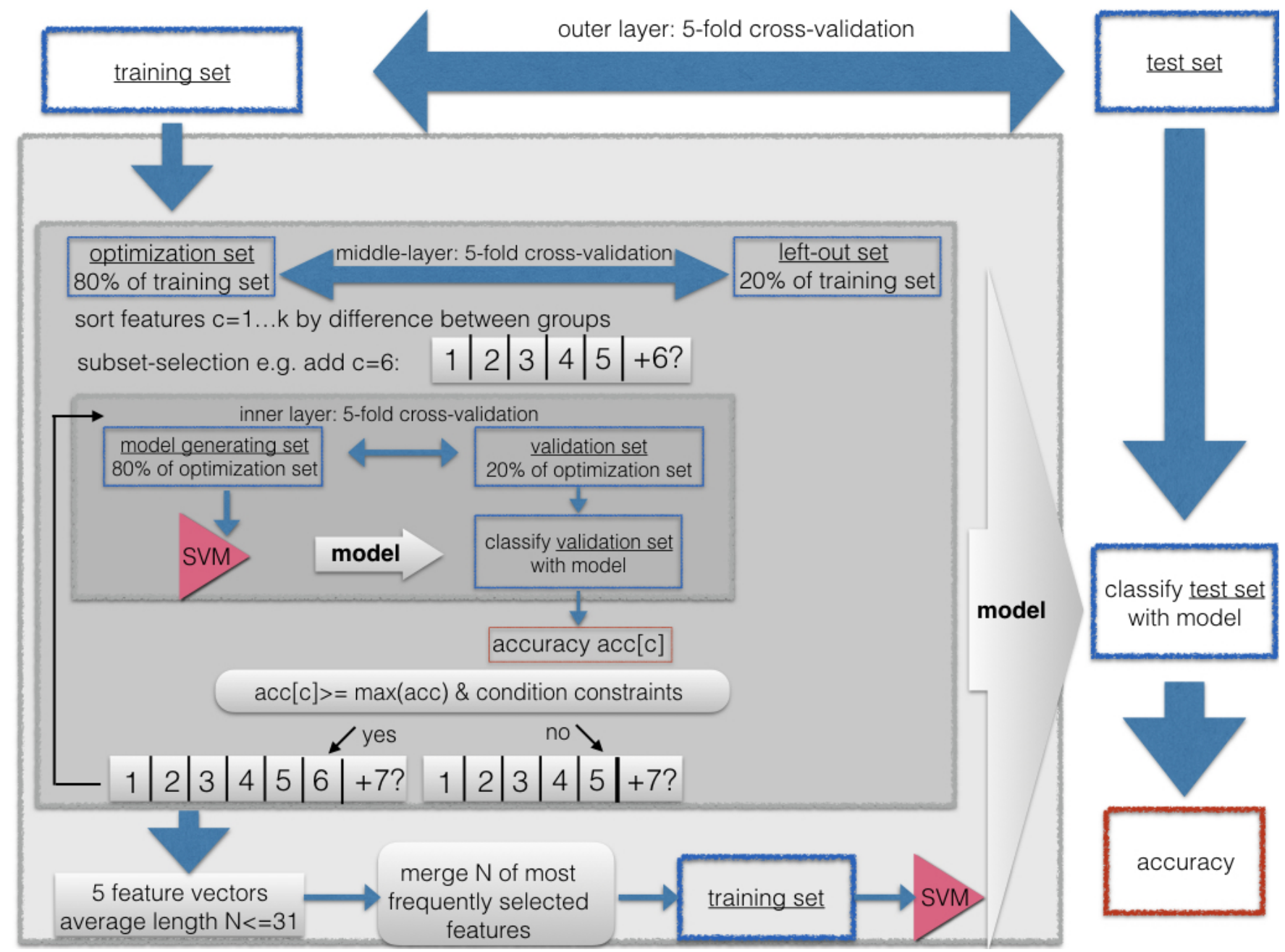

Figure 2: Feature subset selection algorithm in a three-layered cross-validation procedure, in order to shorten and optimize the feature vectors.

age age $<36$ vs. $35<$ age $<63$, age $<36$ vs. $62<$ age, and $35<$ age $<63$ vs. $62<$ age

clinical diagnosis MCI/SCC vs. TLE, MCI/SCC vs. HC, and TLE vs. HC

medication patients with vs. without medication that affects the central nervous system, that is, anti-epileptic or psychiatric drugs

hippocampal pathology patients with vs. without neuroradiological findings on a structural magnetic resonance imaging examination in the memory-region hippocampus, because anomaly in this region is a correlate of TLE and a precursor of dementia

\subsection{Experimental data}

2.7.1 Sample. We examined a total sample of 60 participants, with two EEG recordings from each participant obtained during two sessions, separated by two weeks. Participants with EEG that was of poor quality were excluded beforehand $(\mathrm{N}=3)$. Poor quality of the EEG was defined as less than $4 \mathrm{sec}$ in at least one of the two recordings after excluding segments of $500 \mathrm{~ms}$ according to the automatic data inspection (see Section 2.7.3). Table 1 gives an overview of the demographic characteristics of patients included in the subgroups.

We obtained 8 participants that were younger than 36 years, 27 participants that were up to 62 years old, and 27 participants that were aged 63 years or older.

In order to provide the same amount of data for each participant, data was shortened to the shortest available length across participants, which was 123 seconds. This signal was divided into 6 equal-sized epochs of size 20.5 seconds, which are considered as the input samples to the system. Thus, with two recording sessions, we obtained 12 epochs for each participant.

2.7.2 Data registration. EEG was recorded in two sessions separated by two weeks and took place in the same setting, that is, in a 
Table 1: Sample Overview

\begin{tabular}{lrrr}
\hline & MCI/SCC & TLE & HC \\
\hline N & 27 & 13 & 20 \\
median age & 64 & 48 & 61.5 \\
age range & $48-76$ & $21-66$ & $23-74$ \\
N women & 13 & 9 & 14 \\
N right-handed & 26 & 12 & 18 \\
N CNS medication & 3 & 13 & 0 \\
N hippocampal pathology & 13 & 10 & 9 \\
\hline N= number; MCI= mild cognitive impairment; SCC= \\
subjective cognitive complaints; TLE= temporal lobe \\
epilepsy; l= left; r= right; HC= controls; age= median \\
age; CNS: central nervous system
\end{tabular}

quiet room. Participants were instructed to close their eyes and stay awake. Eyes closed is a condition that allows to reduce artefacts from blinking, and thus variability of the recorded EEG. Recordings lasted for 2-3 min. We used a BrainCap with a 10-20 system and a BrainAmp 16-bit ADC amplifier (Brain Products GmbH, Germany). The sampling rate was $500 \mathrm{~Hz}$. Of the 32 recorded channels, one was used to monitor the lower vertical electrooculogram and one was used to measure electrocardiographic activity. Two were positioned at the earlobes for re-referencing, which was conducted in order to remove the bias of the original reference, which was placed at FCz. Data analysis was conducted for data collected from the remaining 27 electrodes F3, F4, C3, C4, P3, P4, O1, O2, F7, F8, T7, T8, P7, P8, Fz, Cz, Pz, FC1, FC2, CP1, CP2, FC5, FC6, CP5, CP6, TP9, and TP10. Impedances were kept below $10 \mathrm{k} \Omega$.

The two EEG sessions were arranged to take place at the same time of the day. For most participants, this requirement was met by performing EEG within the same time-range around noon (1 pm). This means that we aimed to keep the time difference between the two recordings below three hours. For three participants (HC, SCC, TLEl) the time difference was approximately four hours, for two patients (MCI, TLEr) the time difference was six hours, and for one $\mathrm{HC}$ the time difference was 11 hours.

2.7.3 Data preparation. Data was pre-processed with Brain Vision Analyzer (Version 1.05.0005, Brain Products GmbH). In order to re-reference all channels, a new reference was built by averaging the signal of earlobe electrodes. Butterworth Zero Phase Filters were used for a high-pass filter from $1 \mathrm{~Hz}$ (time constant $0.1592 \mathrm{~s}$, $48 \mathrm{~dB} /$ oct $)$ and an additional notch filter $(50 \mathrm{~Hz})$ was applied.

An automatic artefact detection was carried out in order to exclude highly contaminated datasets. Please note that the automation of this procedure ensures objectivity, which means at the same time that it is reproducible. Nevertheless, the nature and number of artefacts surely depends on the specific recording and participant. Maximal allowed voltage step per sampling point was $50 \mu \mathrm{V}$ (values which exceeded this threshold were marked within a range of $\pm 100 \mathrm{~ms}$ ); maximal allowed absolute difference on an interval of $200 \mathrm{~ms}$ was $200 \mu \mathrm{V}$ and lowest allowed absolute difference during an interval of $100 \mathrm{~ms}$ was $0.5 \mu \mathrm{V}$ (values which exceeded this were marked with a surrounding of $\pm 500 \mathrm{~ms}$ ). The result of this artefact detection was reviewed visually in order to determine whether the
Table 2: Best classification accuracies

\begin{tabular}{lcccc}
\hline & $\begin{array}{c}\text { single } \\
\delta-\gamma\end{array}$ & $\begin{array}{c}\text { single } \\
\alpha-\beta\end{array}$ & $\begin{array}{c}\text { template } \\
\delta-\gamma\end{array}$ & $\begin{array}{c}\text { template } \\
\alpha-\beta\end{array}$ \\
\hline sex & .68 & .65 & .70 & .70 \\
MCI/SCC-TLE & .76 & .77 & .86 & .82 \\
MCI/SCC-HC & .64 & .60 & .66 & .66 \\
TLE-HC & .78 & .75 & .85 & .77 \\
young-middle & .74 & .75 & .74 & .78 \\
young-old & .77 & .79 & .81 & .84 \\
middle-old & .61 & .67 & .61 & .63 \\
hippocampus & .63 & .63 & .63 & .67 \\
CNS drugs & .76 & .75 & .82 & .75 \\
\hline MCI=mild cognitive impairment; SCC=subjective cognitive \\
complaints; TLE=temporal lobe epilepsy; HC=controls; \\
young=age < 36; middle=35 < age < 63; old=62 < age; \\
CNS drugs: intake of drugs affecting the central nervous \\
system; hippocampus=hippocampal pathology
\end{tabular}

automated detection yielded reasonable results and whether poor data quality was due to noise on the reference electrodes, which led to exclusion of the dataset.

The preprocessed data was exported into a generic data format and imported to Matlab ${ }^{\circledR}$ (release R2016b, The Mathworks, Massachusetts, USA).

\section{RESULTS}

Table 2 indicates the best classification results that were obtained across the 16 examined features and the three scenarios of using only the first or the second EEG recording or of pooling the data from both recordings. Interesting results were obtained for the classification of patients with epilepsy vs. other pathological groups, and for the classification of young participants vs. old participants, and the intake of CNS active drugs. Figures 3-6 show the details for the four configurations of using the single epochs with the whole bandwidth (Figure 3) or restricting the bandwidth to $\theta-\beta$ (Figure 4), as well as the use of templates obtained as averages from each 3 single epochs, again with the whole bandwidth (Figure 5) or restricting the bandwidth to $\theta-\beta$ (Figure 6).

According to Table 2 the whole-bandwith analysis of templates yields highest classification accuracies. From Figure 5 we can infer that the most informative features are h, AF, and AR for the identification of patients with TLE and the intake of drugs, while the classification of young vs. old participants was more accurate when based on features such as pCOH, DTF, and ffDTF.

For most features and classifications, the results when using only the first or second EEG or when pooling both sessions are quite comparable. The differentiation between these three scenarios is important, because the EEG varies considerably from day to day, so that the results of single vs. multisession studies on EEG biometrics may lead to different conclusions [27]. However, the two scenarios of using single epochs vs. templates do not only differ with respect to the way the templates were obtained, but also with respect to the sample size that can be used for classification. It seems that the larger sample size in the single-epoch scenarios does not beneficially contribute to the accuracy to an extent that 


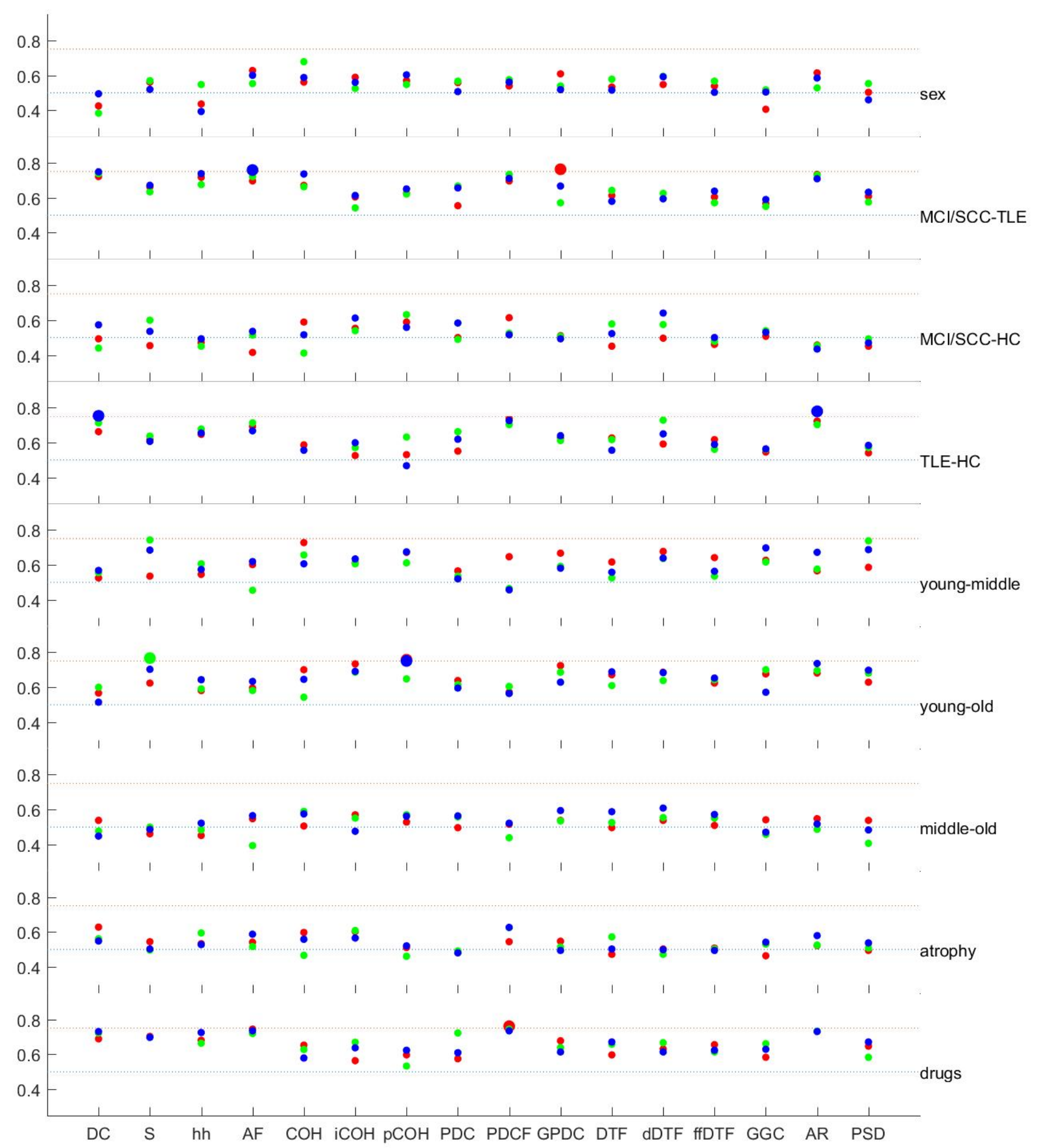

Figure 3: Classification accuracies of the pairwise group classifications for 16 features, in the single epoch scenario for epochs from first EEG only (red), second EEG only (green), both EEGs pooled (blue). Horizontal lines indicate the range between 0.5 and 0.75. Larger markers indicate values $\geq 0.75$. 


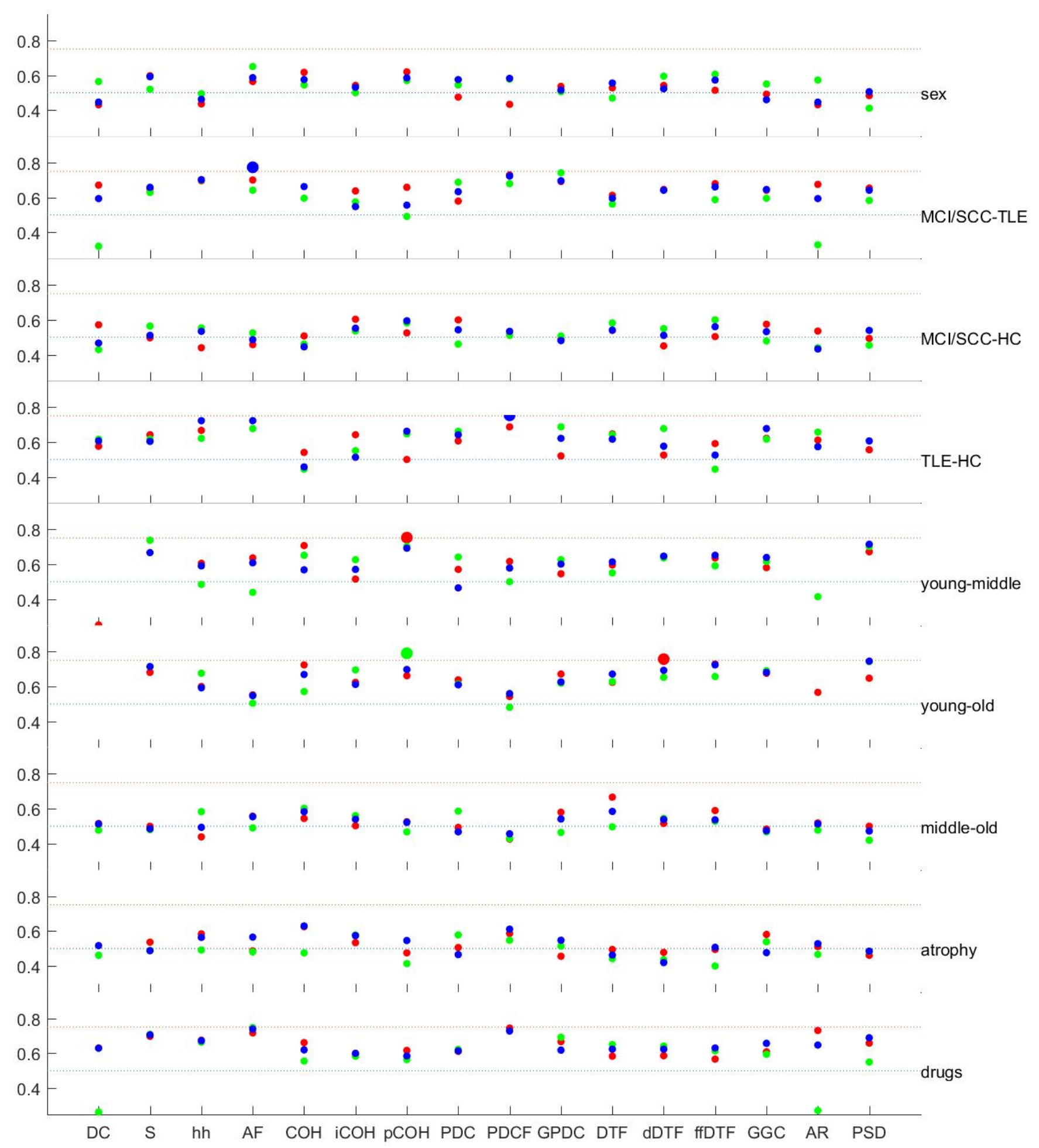

Figure 4: Classification accuracies of the pairwise group classifications for 16 features, in the single epoch scenario when the frequency range was restricted to $\theta, \alpha$, and $\beta$, for epochs from first EEG only (red), second EEG only (green), both EEGs pooled (blue). Horizontal lines indicate the range between 0.5 and 0.75 . Larger markers indicate values $\geq 0.75$. 


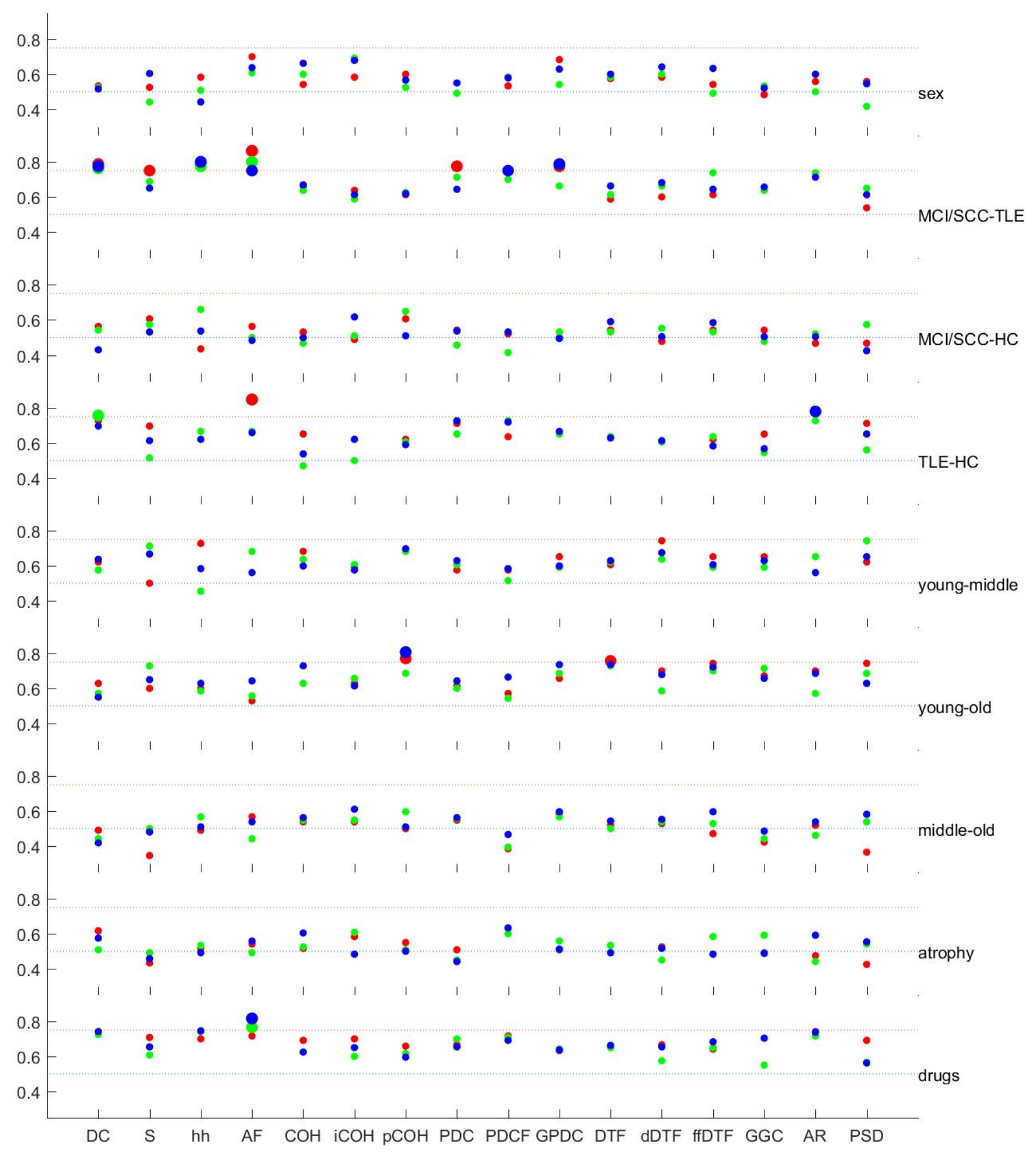

Figure 5: Classification accuracies of the pairwise group classifications for 16 features, in the template scenario for templates from first EEG only (red), second EEG only (green), both EEGs pooled (blue). Horizontal lines indicate the range between 0.5 and 0.75. Larger markers indicate values $\geq 0.75$. 


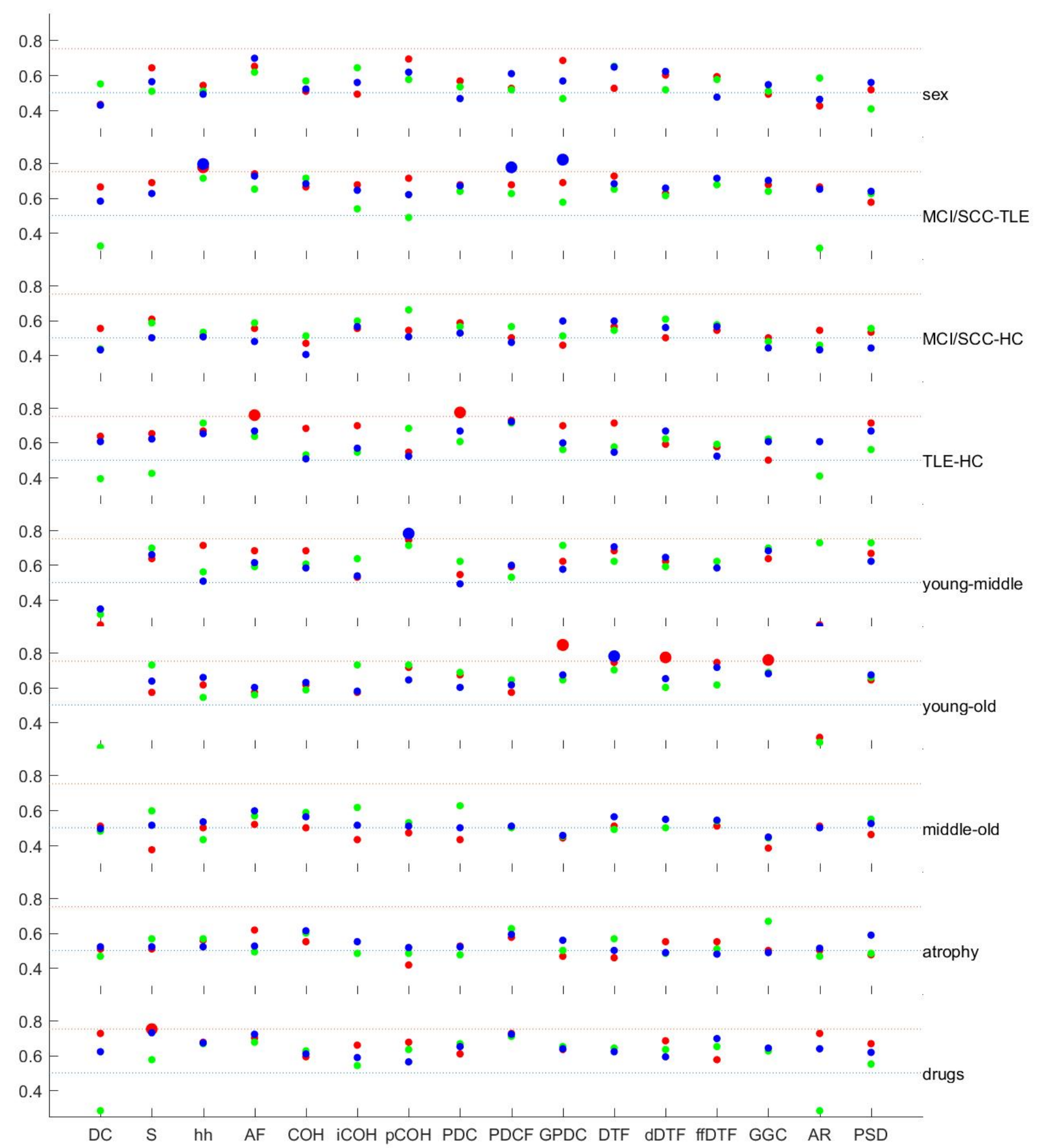

Figure 6: Classification accuracies of the pairwise group classifications for $\mathbf{1 6}$ features, in the in the template scenario when the frequency range was restricted to $\theta, \alpha$, and $\beta$, for templates from first EEG only (red), second EEG only (green), both EEGs pooled (blue). Horizontal lines indicate the range between 0.5 and 0.75 . Larger markers indicate values $\geq 0.75$. 
exceeds the advantage of averaging multiple epochs. Because of the variation of the EEG over time, the average of multiple epochs may be more stable and contain more reliable information than the single epoch data.

The restriction of the frequency range to $\theta-\beta$ may be beneficial when using the system in a sample of healthy subjects. In the presented experimental sample we included neurological populations into our analysis, for which EEG activity in the low $\delta$ frequency band as well as high-frequency activity in the $\gamma$ band could be identifying. As such, the restriction to this range was helpful when differentiating young from old participants. As stated in the introduction, in clinical EEG analyses, the presence of slow activity is a correlate of healthy aging, but may represent also a hint for pathological processes, as well as high-frequency activity is considered to be a marker for epileptogenicity [16].

Among the biomarkers that yielded accuracies above .75, AF and pCOH did so more consistently over the four configurations (single epochs/templates, frequency restriction) than the other markers. Both of these markers strongly emphasize the frequency content of the signal, but in contrast to a classical PSD, these markers take also the multivariate aspect of the EEG into account. In agreement with the current opinion leaders in quantitative EEG research, both, frequency content and spatial interaction of brain activity contain relevant information [10]. Despite a sample size of 60 is quite small, the presented study documents that this information might not only be interesting for clinical purposes such as diagnoses, but it should also be considered in terms of user privacy in EEG biometric systems. Future experiments with larger sample sizes might extend the present work by effective combination of promising features.

\section{CONCLUSIONS}

EEG features and even more so biometric templates can reveal privacy sensitive information about the subject, e.g. the diagnosis of epilepsy or the intake of drugs affecting the central nervous system. Since the sample suffering from TLE overlapped completely with the sample taking the mentioned drugs we cannot disentangle the informative aspect for the classifier. But it is also possible that both aspects contribute to the differentiability of these subjects, since the effect of epilepsy on the EEG is the main reason why EEG is a diagnostic modality in the clinics, and numerous studies document the effect of anti-epileptic drugs on the EEG [26, 30, 47, 66]. Epilepsy is a considerable burden for the affected people, specifically because of the stigma. Moreover, also age is considerably affecting the EEG; a drop in the background dominant frequency from the $\alpha$ frequency band into lower ranges of $\theta$ can be observed both in healthy elderly individuals, and even more so in pathological aging [46]. Thus, the information contained in the biometric templates warrants the use of protective safeguards in EEG biometric systems.

\section{ACKNOWLEDGMENTS}

The authors would like to thank Kevin Butz and Aljoscha Thomschewski for performing the data recording, and Eugen Trinka, Julia Höfler, Judith Dobesberger, Giorgi Kuchukhidze, Markus Leitinger, Margaritha Kirschner, Elisabeth Schmid, Daniela Sinadinoska, Susanne Grinzinger, Martin Scherr, and Wolfgang Staffen for recruitment of patients, and all participants for their time. The presented research was funded by the Austrian Science Fund (FWF): KLI12B00 and by the Paracelsus Medical University Research Support Funds (PMU FFF): A-11/02/004-TRI.

\section{REFERENCES}

[1] G Al-Hudhud, MA Alzamel, E Alattas, and A Alwabil. 2014. Using brain signals patterns for biometric identity verification systems. Computers in Human Behavior 31 (2014), 224-229.

[2] LA Baccalá and K Sameshima. 2001. Partial directed coherence: a new concept in neural structure determination. Biol Cybern 84 (2001), 463-474

[3] LA Baccalá, DY Takahashi, and K Sameshima. 2007. Generalized Partial Directed Coherence. In Proceedings of the 15th International Conference on Digital Signal Processing (DSP); fuly 1-4, Wales, UK, S Sanei, JA Chambers, J McWhirter, Y Hicks, and AG Constantinides (Eds.). IEEE, New York, 162-6.

[4] Garima Bajwa and Ram Dantu. 2016. Neurokey: Towards a new paradigm of cancelable biometrics-based key generation using electroencephalograms. Computers \& Security 62 (2016), 95 - 113. https://doi.org/10.1016/j.cose.2016.06. 001

[5] X. Bao, J. Wang, and J. Hu. 2009. Method of Individual Identification Based on Electroencephalogram Analysis. In 2009 International Conference on New Trends in Information and Service Science. Institute of Electrical and Electronics Engineers ( IEEE ), Beijing, China, 390-393. https://doi.org/10.1109/NISS.2009.44

[6] SL Bressler, CG Richter, Y Chen, and M Ding. 2007. Cortical functional network organization from autoregressive modeling of local field potential oscillations. Stat Med 26 (2007), 3875-85.

[7] K. Brigham and B. V. K. V. Kumar. 2010. Subject identification from electroencephalogram (EEG) signals during imagined speech. In 2010 Fourth IEEE International Conference on Biometrics: Theory, Applications and Systems (BTAS). Washington, DC, USA, 1-8. https://doi.org/10.1109/BTAS.2010.5634515

[8] J Britton, L Frey, J Hopp, P Korb, M Koubeissi, W Lievens, E Pestana-Knight, and E Louis. 2016. Electroencephalography (EEG): An Introductory Text and Atlas of Normal and Abnormal Findings in Adults, Children, and Infants. American Epilepsy Society, Chicago. https://doi.org/10.1002/14651858.CD007272.pub2

[9] CP Brötzner, W Klimesch, M Doppelmayr, A Zauner, and HH Kerschbaum. 2014. Resting state alpha frequency is associated with menstrual cycle phase, estradiol and use of oral contraceptives. Brain Research 19 (2014), 36-44.

[10] E Bullmore and O Sporns. 2009. Complex brain networks: graph theoretical analysis of structural and functional systems. Nat Rev Neurosci 10 (2009), 186-98. https://doi.org/10.1038/nrn2575

[11] John Chuang, Hamilton Nguyen, Charles Wang, and Benjamin Johnson. 2013. I Think, Therefore I Am: Usability and Security of Authentication Using Brainwaves. In Financial Cryptography and Data Security: FC Workshops, USEC and WAHC 2013, Okinawa, Japan, Revised Selected Papers, Andrew A. Adams, Michael Brenner, and Matthew Smith (Eds.). Springer Berlin Heidelberg, Berlin, Heidelberg, 1-16. https://doi.org/10.1007/978-3-642-41320-9_1

[12] P. Cserti, B. Végsö, G. Kozmann, Z. Nagy, F. De Vico Fallani, and F. Babiloni. 2012. Methods to highlight consistency in repeated EEG recordings. IFAC Proceedings Volumes, 8th IFAC Symposium on Biological and Medical Systems 45, 18 (2012), 23 - 27. https://doi.org/10.3182/20120829-3-HU-2029.00078

[13] M DelPozo-Banos, J Alonso, J Ticay-Rivas, and C Travieso. 2014. Electroencephalogram subject identification: a review. Expert Systems with Applications 41,15 (2014), 6537-6554.

[14] M DelPozo-Banos, C Travieso, C Weidemann, and J Alonso. 2015. EEG biometric identification: a thorough exploration of the time-frequency domain. $7 \mathrm{Neural}$ Eng 12 (2015), 056019.

[15] M Eichler. 2006. On the evaluation of information flow in multivariate systems by the directed transfer function. Biol Cybern 94 (2006), 469-82.

[16] B Frauscher, F Bartolomei, K Kobayashi, J Cimbalnik, M A van't Klooster, S Rampp, H Otsubo, Y Höller, J Y Wu, E Asano, J Jr Engel, P Kahane, J Jacobs, and J Gotman. 2017. High-frequency oscillations: The state of clinical research. Epilepsia 58 (2017), 1316-1329. https://doi.org/10.1111/epi.13829

[17] W Gersch and GV Goddard. 1970. Epileptic focus location: spectral analysis method. Science 169 (1970), 701-2.

[18] J Geweke. 1982. Measures of Conditional Linear Dependence and Feedback Between Time Series. 7 Am Stat Assoc 77 (1982), 304-313.

[19] RE Greenblatt, ME Pflieger, and AE Ossadtchi. 2012. Connectivity measures applied to human brain electrophysiological data. Fournal for Neuroscience Methods 207 (2012), 1-16

[20] MJ Kaminskí and KJ Blinowska. 1991. A new method of the description of the information flow in the brain structures. Biol Cybern 65 (1991), 203-210.

[21] M Kaminski, M Ding, WA Truccolo, and SL Bressler. 2001. Evaluating causal relations in neural systems: Granger causality, directed transfer function and statistical assessment of significance. Biol Cybern 85 (2001), 145-57.

[22] J Kennedy and S U Schuele. 2013. Long-term monitoring of brain tumors: when is it necessary? Epilepsia 9 (2013), 50-5. https://doi.org/10.1111/epi.12444 
[23] J. Klonovs, C. K. Petersen, H. Olesen, and A. Hammershoj. 2013. ID Proof on the Go: Development of a Mobile EEG-Based Biometric Authentication System. IEEE Vehicular Technology Magazine 8, 1 (March 2013), 81-89. https://doi.org/10. 1109/MVT.2012.2234056

[24] G Korbakis and P M Vespa. 2017. Multimodal neurologic monitoring. Handb Clin Neurol 140 (2017), 91-105. https://doi.org/10.1016/B978-0-444-63600-3.00006-4

[25] A Korzeniewska, M Maczak, M Kaminskí, KJ Blinowska, and S Kasicki. 2003. Determination of information flow direction among brain structures by a modified directed transfer function (dDTF) method. F Neurosci Methods 125 (2003), 195207.

[26] K Lehnertz and CE Elger. 1997. Neuronal complexity loss in temporal lobe epilepsy: Effects of carbamazepine on the dynamics of the epileptogenic focus. Electroenceph Clin Neurophysiol 103 (1997), 376-80.

[27] E Maiorana, D La Rocca, and P Campisi. 2016. On the Permanence of EEG Signals for Biometric Recognition. IEEE Transactions on Information Forensics and Security 11, 1 (Jan 2016), 163-175. https://doi.org/10.1109/TIFS.2015.2481870

[28] Emanuele Maiorana, Daria La Rocca, and Patrizio Campisi. 2016. Eigenbrains and Eigentensorbrains: Parsimonious bases for EEG biometrics. Neurocomputing 171 (2016), 638 - 648. https://doi.org/10.1016/j.neucom.2015.07.005

[29] S Marple. 1987. Digital Spectral analysis with applications. Prentice Hall, Upper Saddle River, New Jersey.

[30] KJ Meador, A Gevins, PT Leese, C Otoul, and DW Loring. 2011. Neurocognitive effects of brivaracetam, levetiracetam, and lorazepam. Epilepsia 51 (2011), 264-72.

[31] A G Messina, M Wang, M J Ward, C C Wilker, B B Smith, D P Vezina, and N L Pace. 2016. Anaesthetic interventions for prevention of awareness during surgery. Cochrane Database Syst Rev 10, CD007272 (2016). https://doi.org/10. 1002/14651858.CD007272.pub2

[32] F Miraglia, F Vecchio, and P M Rossini. 2017. Searching for signs of aging and dementia in EEG through network analysis. Behav Brain Res 317 (2017), 292-300. https://doi.org/10.1016/j.bbr.2016.09.057

[33] K Mohanchandra, L GM, P Kambli, and V Krishnamurthy. 2013. Using brain waves as new biometric feature for authenticating a computer user in real-time. International Journal of Biometrics and Bioinformatics (IFBB) 7 (2013), 49-57.

[34] VK Murthy. 1963. Estimation of the Cross-Spectrum. Ann Math Statist 34 (1963), 1012-21.

[35] I. Nakanishi, S. Baba, and C. Miyamoto. 2009. EEG based biometric authentication using new spectral features. In 2009 International Symposium on Intelligent Signal Processing and Communication Systems (ISPACS). IEEE, Kanazawa, Japan, 651-654. https://doi.org/10.1109/ISPACS.2009.5383756

[36] G Nolte, O Bai, L Wheaton, Z Mari, S Vorbach, and M Hallett. 2004. Identifying true brain interaction from EEG data using the imaginary part of coherency. Clin Neurophysiol 115 (2004), 2292-307.

[37] M Nuwer. 1997. Assessment of digital EEG, quantitative EEG, and EEG brain mapping: Report of the American Academy of Neurology and the American Clinical Neurophysiology Society. Neurology 49 (1997), 277-292. https://doi.org/ 10.1212/WNL.49.1.277

[38] R Palaniappan. 2006. Electroencephalogram signals from imagined activities: A novel biometric identifier for a small population. In Intelligent Data Engineering and Automated Learning, E Corchado (Ed.), Vol. 4224. Springer-Verlag, Berlin Heidelberg, 604-611. https://doi.org/10.1007/11875581_73

[39] Ramaswamy Palaniappan and Danilo P. Mandic. 2007. EEG Based Biometric Framework for Automatic Identity Verification. The fournal of VLSI Signal Processing Systems for Signal, Image, and Video Technology 49, 2 (2007), 243-250. https://doi.org/10.1007 \/s11265-007-0078-1

[40] Ramaswamy Palaniappan and K.V.R. Ravi. 2006. Improving visual evoked potential feature classification for person recognition using PCA and normalization. Pattern Recognition Letters 27, 7 (2006), 726 - 733. https://doi.org/10.1016/j.patrec 2005.10.020

[41] R. Palaniappan and K. V. R. Ravi. 2003. A new method to identify individuals using signals from the brain. In 2003 Proceedings of the joint Fourth International Conference on Information, Communications and Signal Processing, and the Fourth Pacific Rim Conference on Multimedia, Vol. 3. IEEE, Singapore, Singapore, 1442 1445. https://doi.org/10.1109/ICICS.2003.1292704

[42] CP Panayiotopoulos. 2005. . Bladon Medical Publishing, Oxfordshire, UK.

[43] M. Poulos, M. Rangoussi, V. Chrissikopoulos, and A. Evangelou. 1999. Person identification based on parametric processing of the EEG. In Electronics, Circuits and Systems, 1999. Proceedings of ICECS '99. The 6th IEEE International Conference on, Vol. 1. Pafos, Cyprus, Cyprus, 283-286. https://doi.org/10.1109/ICECS.1999. 812278

[44] K. V. R. Ravi and R. Palaniappan. 2005. Leave-one-out Authentication of Persons Using $40 \mathrm{~Hz}$ EEG Oscillations. In EUROCON 2005 - The International Conference on "Computer as a Tool", Vol. 2. IEEE, Belgrade, Serbia and Montenegro, 1386-1389. https://doi.org/10.1109/EURCON.2005.1630219

[45] F Rosenow, K M Klein, and H M Hamer. 2015. Non-invasive EEG evaluation in epilepsy diagnosis. Expert Rev Neurother 15 (2015), 425-44. https://doi.org/10. 1586/14737175.2015.1025382

[46] P.M. Rossini, S. Rossi, C. Babiloni, and J. Polich. 2007. Clinical neurophysiology of aging brain: From normal aging to neurodegeneration. Prog Neurobiol 83 (2007),
375-400.

[47] MC Salinsky, LM Binder, BS Oken, D Storzbach, CR Aron, and CB Dodrill. 2002. Effects of gabapentin and carbamazepine on the EEG and cognition in healthy volunteers. Epilepsia 43 (2002), 482-90.

[48] W G Sannita. 1990. Quantitative EEG in human neuropharmacology. Rationale, history, and recent developments. Acta Neurol (Napoli) 12 (1990), 389-409.

[49] A Schlögl. 2006. A comparison of multivariate autoregressive estimators. Signal Processing 86 (2006), 2426-2429.

[50] A Schlögl and C Brunner. 2008. BioSig: A Free and Open Source Software Library for BCI Research. Computer 41 (2008), 44-50. Issue 10.

[51] T Schuster and U Kalliauer. 2009. Localizing the focus of epileptic seizures using modern measures from multivariate time series analysis. Diploma-Thesis at the Vienna University of Technology. (2009).

[52] G. K. Singhal and P. RamKumar. 2007. Person Identification Using Evoked Potentials and Peak Matching. In 2007 Biometrics Symposium. IEEE, Baltimore, MD, USA, 1-6. https://doi.org/10.1109/BCC.2007.4430555

[53] H.H Stassen. 1980. Computerized recognition of persons by EEG spectral patterns. Electroencephalography and Clinical Neurophysiology 49, 1-2 (1980), 190 - 194. https://doi.org/10.1016/0013-4694(80)90368-5

[54] Shiliang Sun. 2008. Multitask learning for EEG-based biometrics. In 2008 19th International Conference on Pattern Recognition. IEEE, Tampa, FL, USA, 1-4. https: //doi.org/10.1109/ICPR.2008.4761865

[55] W Szurhaj, M D Lamblin, A Kaminska, H Sediri, and Sociét de Neurophysiologie Clinique de Langue Francaise. 2015. EEG guidelines in the diagnosis of brain death. Neurophysiol Clin 45 (2015), 97-104. https://doi.org/10.1016/j.neucli.2014.11.005

[56] P. Tangkraingkij, C. Lursinsap, S. Sanguansintukul, and T. Desudchit. 2009. Selecting Relevant EEG Signal Locations for Personal Identification Problem Using ICA and Neural Network. In 2009 Eighth IEEE/ACIS International Conference on Computer and Information Science. Shanghai, China, 616-621. https: //doi.org/10.1109/ICIS.2009.156

[57] J Taxidis, B Coomber, R Mason, and M Owen. 2010. Assessing corticohippocampal functional connectivity under anesthesia and kainic acid using generalized partial directed coherence. Biol Cybern 102 (2010), 327-340.

[58] P van Mierlo, E Carrette, H Hallez, K Vonck, D Van Roost, P Boon, et al. 2011. Accurate epileptogenic focus localization through time-variant functional connectivity analysis of intracranial electroencephalographic signals. Neuroimage 56 (2011), 1122-1133.

[59] M J van Putten and J Hofmeijer. 2016. Multimodal neurologic monitoring. F Clin Neurophysiol 33 (2016), 203-10. https://doi.org/10.1097/WNP.0000000000000272

[60] O Vysata, J Kukal, A Prochazka, L Pazdera, J Simko, and M Valis. 2014. Agerelated changes in EEG coherence. Neurologia I Neurochirurgia Polska 48 (2014), 35-38.

[61] Y Wada, Y Nanbu, R Kadoshima, ZY Jiang, Y Koshino, and T Hashimoto. 1996. Interhemispheric EEG coherence during photic stimulation: sex differences in normal young adults. Int 7 Psychophysiol 22 (1996), 45-51.

[62] Y Wada, Y Takizawa, ZY Jiang, and N Yamaguchi. 1994. Gender differences in quantitative EEG at rest and during photic stimulation in normal young adults. Clin Electroencephalogr 25 (1994), 81-5. Issue 2.

[63] S. Yang and F. Deravi. 2017. On the Usability of Electroencephalographic Signals for Biometric Recognition: A Survey. IEEE Transactions on Human-Machine Systems PP, 99 (2017), 1-12. https://doi.org/10.1109/THMS.2017.2682115

[64] A. Yazdani, A. Roodaki, S. H. Rezatofighi, K. Misaghian, and S. K. Setarehdan. 2008. Fisher linear discriminant based person identification using visual evoked potentials. In 2008 9th International Conference on Signal Processing. IEEE, Beijing, China, 1677-1680. https://doi.org/10.1109/ICOSP.2008.4697459

[65] Seul-Ki Yeom, Heung-Il Suk, and Seong-Whan Lee. 2013. Person authentication from neural activity of face-specific visual self-representation. Pattern Recognition 46, 4 (2013), 1159 - 1169. https://doi.org/10.1016/j.patcog.2012.10.023

[66] HP Zaveri, SM Pincus, II Goncharova, EJ Novotny, RB Duckrow, DD Spencer, H Blumenfeld, and SS Spencer. 2010. Background intracranial EEG spectral changes with anti-epileptic drug taper. Clin Neurophysiol 121 (2010), 311-7. https://doi.org/10.1016/j.clinph.2009.11.081 